\title{
Ambulante Ernährungstherapie
}

\begin{abstract}
— Für viele Krebspatienten ist in der ambulanten Nachversorgung die individuelle Ernährungstherapie für den Krankheits- und Therapieverlauf besonders entscheidend. Um die ernährungsmedizinische Nachsorge zu optimieren, setzen seca als Weltmarktführer im Bereich medizinischer Messsysteme und Waagen, und B. Braun TravaCare als Experte für die heimparenterale Ernährung, auf den Einsatz validierter BIA-Technologie. Zukünftig werden Ernährungsspezialisten von B. Braun TravaCare den Gesundheitsund Ernährungszustand der Patienten mithilfe des seca mBCA 525 sekundenschnell bestimmen und die Therapie individuell gestalten.
\end{abstract}

"Die ambulante Ernährungstherapie im heimischen Umfeld des Patienten ist eine pflegerisch extrem anspruchsvolle Arbeit. Unser Ziel ist es, Versorgung auf höchstem Qualitätsniveau zu bieten", so Dr. Dr. Peter Adamczyk, einer der Geschäftsführer von B. Braun TravaCare. Die nachweisbare Präzisi- on der Parameter und die einfache mobile Anwendung seien u.a. ausschlaggebend für die Entscheidung für den seca mBCA 525. „Durch den Einsatz der mobilen BIA-Messstation wollen wir die Therapie optimieren, das Monitoring und die Lebensqualität der Patienten verbessern und damit das Niveau unser Leistung weiter steigern", fügt Adamczyk hinzu. Besonders in der Onkologie und zur Vermeidung von Mangelernährung ist das messsystem geeignet, um den Gesundheits- und Ernährungszustand der Patienten präzise zu bestimmen. Die Präzision der BIA-Parameter wurde in einer multizentrischen Studie über verschiedene Ethnien gegen den jeweiligen Goldstandard validiert. Geliefert wird eine vollständige Analyse der Körperzusammensetzung für Diagnose und Therapie. Die Messmatte misst alle erforderlichen Parameter eines liegenden Patienten, wie Fettmasse, Muskelmasse und Körperwasser, in nur 30 Sekunden und

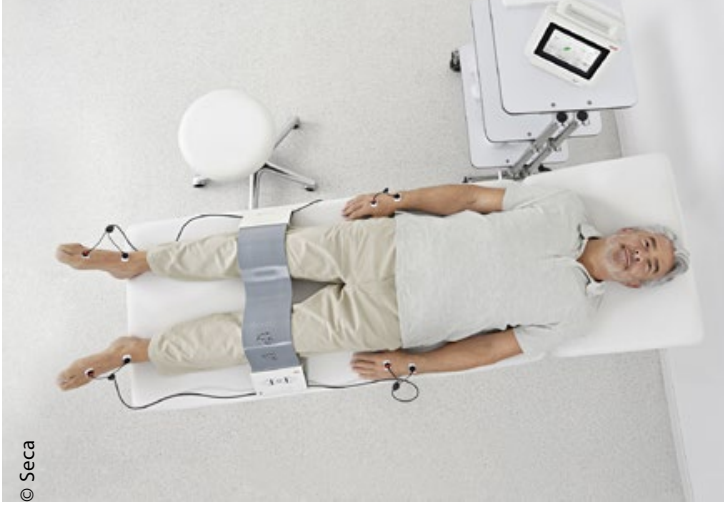

überträgt die Werte über WLAN an den Touchscreen-Monitor und in die digitale Patientenakte. Die Matte wird von einem eigenen Akku gespeist, der per Induktion geladen wird - ein Garant für hohe Mobilität. Der Datenspeicher des Monitors kann mehr als 100.000 Messungen erfassen und somit zahlreiche Patienten und Therapieverläufe jahrelang zuverlässig und ortsunabhängig dokumentieren. Die übersichtliche Darstellung aller Parameter auf dem Monitor sowie optional auf einem einseitigen Ergebnisausdruck unterstützt die Diagnose des Arztes und erleichtert das Patientengespräch.

www.seca.com

\section{Unschöne Narben vermeiden}

\section{Prävention und Therapie pathologischer Narben}

- Hypertrophe Narben und Keloide sind kein rein kosmetisches Problem, sondern können mit Juckreiz, Spannungsgefühl, Schmerzen und Funktionseinschränkungen einhergehen. Für Patienten mit erhöhtem Risiko pathologischer Narbenbildung und zur Behandlung überschießender Narben eignet sich ein Gel oder Patch mit Zwiebelextrakt und Allantoin.

Exzessive Bildung von Narbengewebe kann nach tiefen Verletzungen der Haut in Abhängigkeit von individueller Disposition (Hormone, Genetik), Lokalisation, chronischen Entzündungen (wie Akne), bei vermehrtem Zug auf die Wundränder oder verzögerter Wundheilung auftreten, erklärte PD Gerd Gauglitz, Klinik und Poliklinik für Dermatologie und Allergologie, Ludwig-MaximiliansUniversität München. Zur Behandlung von Keloiden und hypertrophen Narben empfehlen die aktuellen deutschen und internationalen Leitlinien intraläsionale Kortikosteroide und Kryotherapie teilweise in Kombination. Neuere Verfahren wie Farbstofflaser,
5-Fluoruracil und fraktionierte Laser werden je nach Indikation in den aktualisierten internationalen Leitlinien positiv bewertet.

Das in der täglichen Praxis häufig angewandte Narbentopikum Contractubex ${ }^{\circledR}$ mit Extractum Cepae, Heparin und Allantoin habe seine Wirksamkeit auf die Narbenheilung in verschiedenen Studien belegt, so Gauglitz. Der Zwiebelextrakt wirke anti-proliferativ und anti-inflammatorisch auf Fibroblasten und hemme die in überschießenden Narben gesteigerte Kollagenproduktion. In einer prospektiven randomisierten Studie an 65 Kindern mit Operationen im Thoraxbereich konnte gezeigt werden, dass eine frühzeitige Behandlung ( 3 Wochen nach OP, $2 x$ tgl.) mit dem Zwiebelextrakt in den ersten sechs Monaten zu deutlich kleineren Narben führte; überschießende Narben entwickelten sich seltener als in der Vergleichsgruppe ohne Behandlung. Eine Beobachtungsstudie an 1268 Patienten ergab eine signifikante Verbesserung der Narbenqualität durch den Zwiebelextrakt bei Patienten mit Zustand nach OP, Schnitt- und Schürfwunden sowie Verbrennungen. Die Anwendung des Narbengels wurde in die internationalen Leitlinien zur Behandlung von überschießenden Narben aufgenommen. Hier werde es für jüngere, hypertrophe Narben und zur Prävention bei Patienten mit erhöhtem Risiko der pathologischen Narbenentstehung empfohlen. Seit kurzem steht mit dem Contractubex ${ }^{\circledast}$-Intensivpatch (Extractum Cepae, Allantoin) eine Narbentherapie für die Nacht mit okklusivem Effekt zur Verfügung. Die dreimonatige Anwendung des Patches auf hypertrophen Narben zeige eine signifikante Abflachung der Narbendicke sowie eine deutliche Verbesserung von Juckreiz und Schmerzen, so Gauglitz. Prophylaktisch könne mit der postoperativen Anwendung kurz nach Fadenzug begonnen werden.

(sp)

Mittagsseminar „Update Narbenprävention und Narbentherapie"; München, 25. Juli 2016 (Veranstalter: Merz Pharmaceuticals $\mathrm{GmbH}$ ) 